This item was submitted to Loughborough's Research Repository by the author.

Items in Figshare are protected by copyright, with all rights reserved, unless otherwise indicated.

\title{
Performance estimation of 2-sliding mode controllers for chatter reduction in linear systems
}

\section{PLEASE CITE THE PUBLISHED VERSION}

http://dx.doi.org/10.1049/iet-cta.2011.0685

PUBLISHER

(C) IET

VERSION

AM (Accepted Manuscript)

LICENCE

CC BY-NC-ND 4.0

\section{REPOSITORY RECORD}

O'Toole, Michael D., Kaddour Bouazza-Marouf, and David Kerr. 2019. "Performance Estimation of 2-sliding Mode Controllers for Chatter Reduction in Linear Systems". figshare. https://hdl.handle.net/2134/12096. 
This item was submitted to Loughborough's Institutional Repository (https://dspace.lboro.ac.uk/) by the author and is made available under the following Creative Commons Licence conditions.

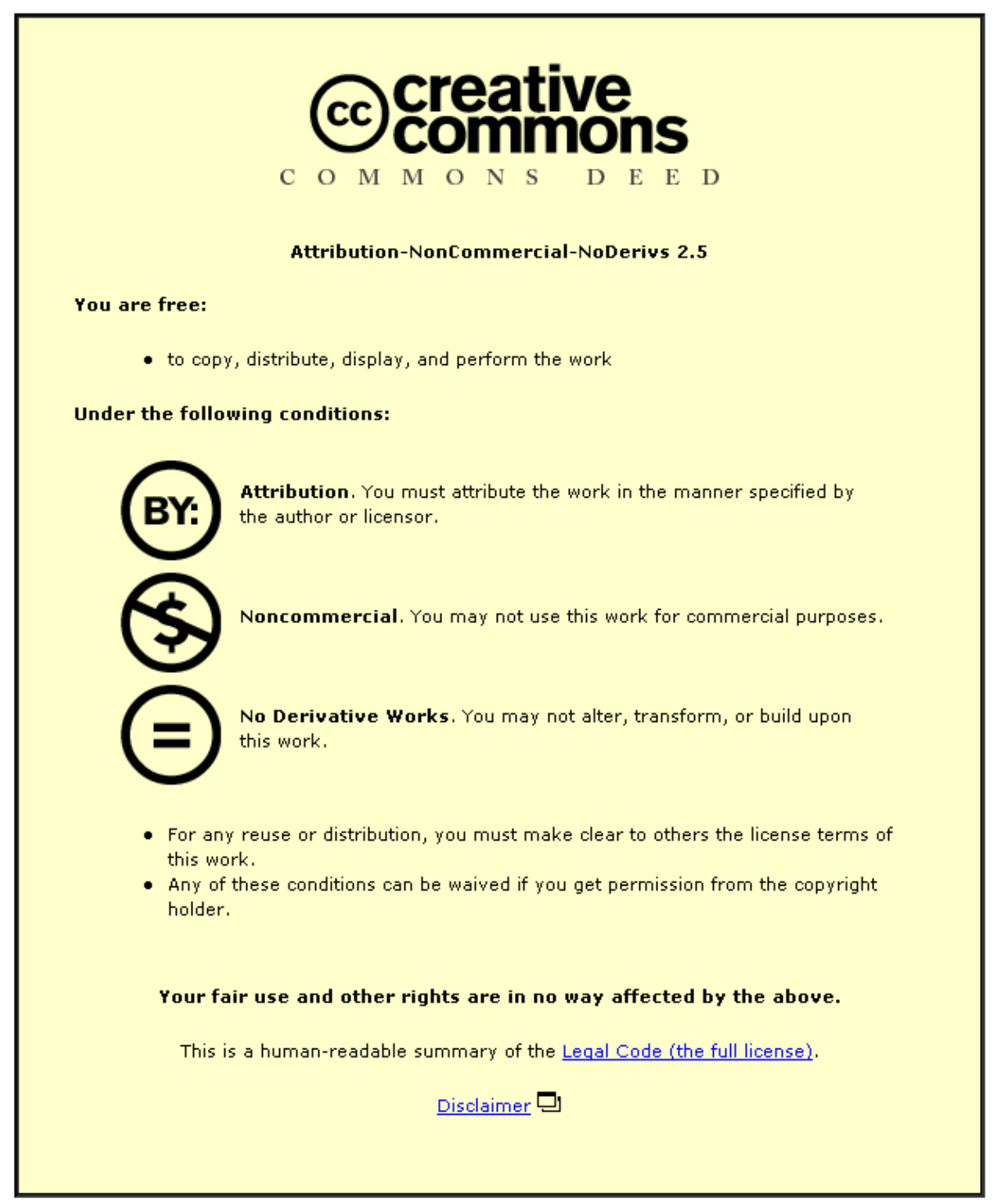

For the full text of this licence, please go to: http://creativecommons.org/licenses/by-nc-nd/2.5/ 


\title{
Performance Estimation of 2-Sliding Mode Controllers for Chatter Reduction in Linear Systems
}

\author{
M. D. O'Toole, K. Bouazza-Marouf* and D. Kerr, \\ Wolfson School of Mechanical \& Manufacturing Engineering, \\ Loughborough University, \\ Leicestershire, UK, LE11 3TU \\ *K.Bouazza-Marouf@lboro.ac.uk
}

\begin{abstract}
Sliding mode control is a simple and powerful technique for the robust control of uncertain systems. However, despite theoretical promise, real systems using sliding mode controllers have a tendency to chatter at high frequencies. Second order, or 2-Sliding mode controllers, can be used to suppress chatter while retaining the desirable properties of a classical sliding mode controller. These more complex controllers increase the number of parameters in the system. The affect of these new parameters on system performance is unclear, and this can make it difficult to tune the controllers to meet a desired performance criteria. This paper addresses the problem of estimating the performance of a system controlled by 2-sliding mode controllers. Describing functions are proposed to estimate chattering amplitude and settling time, and a new method is presented, based on invariant ellipsoid sets, to predict the maximum output bound of the system. The aim is assist in producing a systematic approach for practitioners wishing to directly apply sliding mode controllers with chatter suppressing properties to real applications.
\end{abstract}

\section{Introduction}

Sliding mode control is a simple control technique that has proven a valuable tool for the control of systems with uncertainty. It is invariant to matched uncertainty and disturbances provided that the system has relative degree one with respect to the output sliding variable $[9,16]$. In any real system however, uncertain and un-modelled fast-acting parasitic dynamics will affect this structure by raising the relative degree. This change causes the system to exhibit high frequency, self-sustaining oscillations or limit cycles about the sliding surface. This effect is referred to as chattering. 
Chattering is a well-known phenomenon in sliding mode control. The damage, inefficiency, and wear that chattering can cause is a severe hindrance to the use of this control technique in real-world applications. The need to resolve chatter in practical systems has directed research into controllers that embody the qualities of sliding mode control (robustness, etc.), but which can also suppress chatter. These controllers are invariably more complex than the classical sliding mode controller, and introduce new parameters, or degrees-of-freedom, to the system structure. The designer must know how the choice of these parameters will change the system behaviour, and how to tune the controller to meet a desired performance specification.

Higher order sliding mode controllers are a class of controllers that preserve the properties of classical sliding mode control, while reducing chattering. In this paper, we will consider second order sliding mode controllers, called 2Sliding mode controllers, which are a sub-class of this group. Classical sliding mode control drives and maintains the output sliding variable at zero. In this state, the system is said to be in a sliding mode or motion, and the dynamics become invariant to uncertainty and disturbances under certain conditions [9]. In contrast, 2-Sliding mode controllers drive the output sliding variable and its first derivative with respect to time to zero, and maintains them at zero thereafter. This allows for an increased relative degree in the system, and mitigates the effects of chattering.

2-Sliding mode controllers are more complex algorithms than classical sliding mode controllers, and introduce new design parameters that must be chosen or tuned. The method of tuning these parameters becomes vital in delivering potential performance improvements. A methodology is presented in [8] to design higher order sliding mode controllers based on an analogous optimal control problem for a chain of perturbed integrators. The authors through their analysis are able to create relatively straightforward rules to select design parameters to optimally enter sliding motion (output sliding variable equal to zero) and guarantee stability. A self-tuning approach is proposed in [18] for finite time stabilisation of systems with unknown but bounded uncertainty. The hard non-linearities in the 2-sliding mode controller are smoothed by a 'soft' bipolar sigmoid function. The parameters of the new controller are tuned online to vary the smoothing of the function, in order to reduce the chattering and steady-state error. Similarly, an adaptive 2-sliding mode control scheme is presented in [13]. The scheme consists of an adaptive component to optimally enter sliding motion for the nominal system (i.e. without uncertainties), and adaptive relay gain, i.e. gain of the non-linearity, to accommodate unknown but bounded uncertainty, and reduce the control effort and resultant chattering. However, the above works do not consider explicitly the effect of the 2-sliding mode controller on the resultant chatter. Nor do they provide tuning methods for the different types of 2-sliding mode algorithms. It is important to be able to transparently approximate performance for a range of possible specification metrics and provide knowledge of whether they will be met or not.

In this paper, we propose procedures for estimating the performance of systems controlled by 2 -sliding mode controllers with chatter suppressing proper- 
ties. The aim is to create tools to assist in the design and tuning of 2-sliding mode controllers for chatter suppression in real-systems. Three performance metrics are considered; they are chattering amplitude, settling time, and maximum output bound (overshoot). These performance metrics are well known to classical control systems design, and are integral to many control system specifications.

The paper has the following structure: In section two, three commonly used 2 -sliding mode controllers are surveyed. They are the twisting controller [10], the generalized sub-optimal controller [2], and the homogeneous controller [11, 12]. In section three, a method for obtaining settling time using describing functions is briefly reviewed, and new functions are introduced for two of the controllers. When a damping term in each function is set to zero, the describing functions become those published in $[5,4]$. In section four, a new procedure for estimating the maximum output of switching systems is proposed. The method uses successive invariant ellipsoid sets to enclose the state trajectory of the system. The application of this procedure to all three 2-sliding mode controllers is discussed. In section five, an example system is presented, and simulated results obtained to illustrate the concepts in this paper.

\section{2-Sliding Mode Controllers}

The standard sliding mode controller assumes the system with respect to the output sliding variable has relative degree one. Higher order sliding modes allow this condition to be relaxed. For simplicity, we reserve our discussion to higher order sliding mode controllers designed for systems with relative degree two referred to as 2-sliding mode controllers. One of the most useful features of 2sliding mode controllers is their chatter suppression properties. When properly tuned, they can be used to suppress chatter in a closed-loop system without compromising tracking accuracy or robustness. We review three of the most commonly used 2-sliding mode controllers, in addition to their stability conditions, in this section.

We first define some preliminary notation. Introducing an $n^{\text {th }}$-order singleinput-single-output smooth dynamical system with a control input $u(t)$,

$$
\dot{z}=\mathbf{A} z+\mathbf{B} u(t), \quad \sigma=S z,
$$

where $\mathbf{A} \in \mathbb{R}^{n \times n}, \mathbf{B} \in \mathbb{R}^{n}$, and $z \in \mathbb{R}^{n}$ is the state vector. The term $\sigma$ is the output sliding variable. The sliding surface is defined by the vector $S \in \mathbb{R}^{n}$ and is determined by the designer (see [9]). The system in equation (1) is extended to include first-order parasitic dynamics on the actuator (input) and sensor (output) side,

$$
\left[\begin{array}{c}
\dot{z} \\
\mu_{s} \dot{x} \\
\mu_{a} \dot{\tilde{u}}
\end{array}\right]=\left[\begin{array}{ccc}
\mathbf{A} & \mathbf{0}_{n \times n} & \mathbf{B} \\
\mathbf{I}_{n} & \mathbf{I}_{n} & \mathbf{0}_{n} \\
\mathbf{0}_{1 \times n} & \mathbf{0}_{1 \times n} & -1
\end{array}\right]\left[\begin{array}{c}
z \\
x \\
\tilde{u}
\end{array}\right]+\left[\begin{array}{l}
0 \\
0 \\
1
\end{array}\right] u(t), \quad \sigma=S x,
$$

where $\tilde{u}$ is the actuator state-variable, $x \in \mathbb{R}^{n}$ the sensor state-variable, and $\mu_{a}, \mu_{s}$ their respective time-constants. It is assumed that all fast-acting parasitic 
dynamics present in any real-system can be adequately approximated by the extended system. The open-loop system in equation (2) can redefined as the second derivative of the output switching function, i.e.,

$$
\ddot{\sigma}=h(z, x, \tilde{u})+g(z, x, \tilde{u}) u(t)
$$

where $h(z, x, \tilde{u})$ and $g(z, x, \tilde{u})$ are smooth functions that can be determined from input-output relations, i.e. $h(z, x, \tilde{u})=|\ddot{\sigma}|_{u=0}$, and $g(z, x, \tilde{u})=(\partial / \partial u) \ddot{\sigma}$. Both functions are assumed to be bounded by the constants $K_{M}, k_{m}, C>0$ such that,

$$
0<K_{m}<\frac{\partial \ddot{\sigma}}{\partial u} \leq K_{M}, \quad|\ddot{\sigma}|_{u=0} \leq C .
$$

From these bounds, a differential inclusion is derived,

$$
\ddot{\sigma} \in[-C, C]+\left[K_{M}, K_{m}\right] u(t)
$$

The differential inclusion is used in the stability conditions for the sliding mode controllers.

The 2-sliding mode controllers are as follows:

1) The twisting controller [10]:

$$
u(\sigma)=-\left(r_{1} \operatorname{sgn}(\sigma)+r_{2} \operatorname{sgn}(\dot{\sigma})\right),
$$

where $r_{1}$ and $r_{2}$ are design parameters. The controlled system is stable if $0<$ $r_{2}<r_{1}$ and

$$
\begin{gathered}
\left(r_{1}+r_{2}\right) K_{m}-C>K_{M}\left(r_{1}-r_{2}\right)+C \\
\left(r_{1}-r_{2}\right) K_{m}>C .
\end{gathered}
$$

2) The generalized sub-optimal controller [2]:

$$
u(\sigma)=-r_{1} \operatorname{sgn}\left(\sigma-r_{2} \sigma_{M}\right)
$$

where $\sigma_{M}$ is the previous extremal value of the output sliding variable. The advantage of this controller is that no intermediate differentiator is required to calculate $\dot{\sigma}$, and so the algorithm is simpler and less susceptible to noise than the other 2-sliding mode controllers. The stability conditions are,

$$
r_{1}>C / K_{m}, \quad r_{2}>\frac{2 C+(1-\beta) K_{M} r_{1}}{(1+\beta) K_{m} r_{2}}
$$

3) The homogeneous controller $[11,12]$ :

$$
u(\sigma)=-r_{1} \operatorname{sgn}\left(\dot{\sigma}+r_{2}|\sigma|^{1 / 2} \operatorname{sgn}(\sigma)\right) .
$$

The stability condition is $r_{1} K_{m}-C>r_{2}^{2} / 2$. 


\section{Chattering Amplitude and Settling Time}

This section first reviews a method to approximate settling time and chattering amplitude using describing functions (sometimes referred to as harmonic linearisation) $[17,1,14]$. The method is well suited to controllers that are discontinuous, and systems with a chattering steady-state response. We then derive new describing functions for two of the 2 -sliding mode controllers. These functions can be used to compute an instantaneous damping and frequency term, which in turn, can be used to calculate settling time and chattering amplitude.

A describing function is a complex first order Fourier-series approximation of the controller, that assumes a diminishing oscillatory feedback. As the function is a first order approximation, the following assumptions must be valid:

Assumption 1: An open-loop system with sliding variable as output, has a frequency response equivalent to a low-pass filter. if the system does not behave as a low-pass filter, then the higher-order terms in the Fourier approximation will diminish the accuracy of the method.

Assumption 2: The behaviour of the sliding variable about the sliding surface can be approximated by the function $\sigma(t)=a(t) \sin (\theta(t))$.

Neither need be precisely true, however, the closer the system is to meeting these assumptions, the more accurate the resulting analysis. In practice, these assumptions can apply to many useful linear models and common real-world systems. The assumptions are thus not overly restrictive. However, it is worth a note of caution when applying the describing function method, that the validity of the results depends on the extent the assumptions are met.

Denote the characteristic equation of a system with output $\sigma$ as

$$
A(p) \sigma(t)+B(p) F(\sigma, p \sigma)=0,
$$

where $p$ is the Laplace variable, $A(p), B(p)$ are the linear components of the system and $F(\sigma, p \sigma)$ the non-linear controller. We assumed that the behaviour of the output sliding variable can be approximated by the function $\sigma(t)=$ $a(t) \sin (\theta(t))$. Then, a suitable first order approximation of the time-varying amplitude and time-varying phase can be obtained by,

$$
a(t)=a(0) e^{\zeta t} \Rightarrow \frac{d a}{d t}=\zeta(t) a(t) \quad \text { and } \quad \frac{d \theta(t)}{d t}=\omega(t),
$$

where $\zeta$ and $\omega$ are pseudo-static terms that vary slowly with time $(\dot{\zeta} \approx 0, \dot{\omega} \approx 0)$. It follows that

$$
\begin{aligned}
& \sigma(t) \approx \tilde{\sigma}(t)=a(t) \sin (\theta(t)) \\
& \dot{\sigma}(t) \approx p \tilde{\sigma}(t)=a(t)(\zeta(t) \sin (\theta(t))+\omega(t) \cos (\theta(t))) .
\end{aligned}
$$

Thus, the first-order Fourier series of the non-linear function is

$$
F(\sigma, p \sigma)=N(a, \zeta, \omega) \sigma(t)=\frac{1}{a}[P(a, \zeta, \omega)+j Q(a, \zeta, \omega)] \sigma(t),
$$




$$
\begin{aligned}
& P=\frac{1}{\pi} \int_{0}^{2 \pi} F[\tilde{\sigma}, p \tilde{\sigma}] \sin (\theta(t)) d \theta \\
& Q=\frac{1}{\pi} \int_{0}^{2 \pi} F[\tilde{\sigma}, p \tilde{\sigma}] \cos (\theta(t)) d \theta .
\end{aligned}
$$

An expression for the damping $\zeta$ and frequency $\omega$, in terms of amplitude $a$, is obtained by substituting the describing function into (9) and by setting the Laplace variable to $p=\zeta+j \omega$. Choosing $\zeta=0$ and solving for $\omega$ and $a$ gives the chattering frequency and chattering amplitude respectively. The time for the system to settle from initial oscillation $a_{0}$ amplitude to some amplitude $a_{k}$ is given by

$$
t_{s}=-\int_{a_{0}}^{a_{k}} \frac{1}{a \zeta(a)} d a
$$

which is derived from (10). The describing functions for the twisting controller and generalized sub-optimal controller are as follows:

1. Twisting controller:

$$
N(a, \zeta, \omega)=\frac{4}{\pi a}\left(r_{1}-\frac{r_{2}}{\sqrt{1+(\omega / \zeta)^{2}}}-j \frac{r_{2} \omega}{\zeta \sqrt{1+(\omega / \zeta)^{2}}}\right) .
$$

When $\zeta=0$ we obtain the result presented in [5].

2. Generalized sub-optimal controller:

$$
\begin{aligned}
N(a, \zeta, \omega) & =\frac{4 r_{1}}{\pi a}\left(\sqrt{1-\Sigma^{2}}+j \Sigma\right), \\
\text { where } \quad \Sigma & =\frac{r_{2}(1-\zeta / \omega)}{e^{\zeta \pi /(4 \omega)}-\sqrt{2} r_{2} \zeta / \omega} .
\end{aligned}
$$

When $\zeta=0$ we obtain the result presented in [6].

The describing function for the twisting controller is derived directly from the Fourier series. The describing function for the generalized suboptimal controller is similarly derived from the Fourier series and an approximation of the phase angle at switching. Switching of the controller occurs when $\sigma=r_{2} \sigma_{M}$. Recalling that $\sigma \approx a(t) \sin (\theta(t))$ and $a(t)=a(0) e^{\zeta t}$ and assuming the angle at switching is $\theta_{s} \approx \omega t$, i.e. $\omega(t)$ changes slowly over the period, then (10) can be rearranged such that the phase angle at switching is the solution to the equality,

$$
\sin \theta_{s}=r_{2} e^{\zeta\left(\pi / 2-\theta_{S}\right) / \omega} \approx \frac{r_{2}(1-\zeta / \omega)}{e^{\zeta \pi /(4 \omega)}-\sqrt{2} r_{2} \zeta / \omega},
$$

where the right-hand term is derived using the Taylor expansion. Any function for the homogeneous controller requires a solution to,

$$
(\zeta / \omega) \sin \theta_{s}+\cos \theta_{s}+\frac{r_{2}}{\omega \sqrt{a}} \sqrt{\sin \theta_{s}}=0 .
$$


Local linearisation and approximations can be used to obtain a closed-form solution of this equation. However, producing adequate approximations, i.e. within $5 \%$ of the actual solution, results in large and unwieldy functions. Thus, we do not advocate the this method for the homogeneous controller.

\section{Maximum Output Bound}

In this section, a unified approach to establishing maximum output bound is proposed for systems controlled by the 2-sliding mode controllers in section 2. The approach computes a sequential series of invariant ellipsoid sets $[3,7$, 15] which enclose the trajectory of the state-variable in the state-space. The geometric properties of the ellipsoids are then used to obtain the maximum output bound. We first introduce a new theorem that determines a set of linear matrix inequalities that must be satisfied for the ellipsoid sets to be invariant and to contain the state trajectory. The theorem is then used to construct a procedure for each of the 2-sliding mode controllers to find maximum output bound for some choice of tuning parameters.

Theorem 1. Suppose a system with state variable $y \in \mathbb{R}^{n}$ is governed by a sequential series of dynamic equations of the form

$$
\begin{aligned}
\dot{y} & =\mathcal{A}_{i} y-\mathcal{B}_{i} \rho_{i}, \\
\dot{y} & =\mathcal{A}_{i+1} y-\mathcal{B}_{i+1} \rho_{i+1},
\end{aligned}
$$

where $\mathcal{A} \in \mathbb{R}^{n \times n}, \mathcal{B} \in \mathbb{R}^{n}$, and $\rho \in \mathbb{R}^{1}$. Switching from the $i^{\text {th }}$ to the $(i+1)^{\text {th }}$ dynamic equation occurs when the trajectory of $y$ crosses the manifold $\mathcal{C}_{i} y=\beta_{i}$, where $\mathcal{C}_{i} \in \mathbb{R}^{1 \times n}$. If the trajectory, while governed by the $i^{\text {th }}$ dynamic equation, starts within or enters a set bounded by an ellipsoid

$$
\left(y-\mathcal{A}_{i}^{-1} \mathcal{B}_{i} \rho_{i}\right)^{T} P_{i}\left(y-\mathcal{A}_{i}^{-1} \mathcal{B}_{i} \rho_{i}\right) \leq 1,
$$

then after crossing the manifold $\mathcal{C} y=\beta_{i}$, the trajectory, while governed by the $(i+1)^{\text {th }}$ dynamic equation, will be enclosed by an ellipsoid

$$
\left(y-\mathcal{A}_{i+1}^{-1} \mathcal{B}_{i+1} \rho_{i+1}\right)^{T} P_{i+1}\left(y-\mathcal{A}_{i+1}^{-1} \mathcal{B}_{i+1} \rho_{i+1}\right) \leq 1,
$$

provided the following conditions are satisfied:

$$
\begin{array}{cc}
(1) & \mathcal{A}_{i}^{T} \bar{P}_{i}+\bar{P}_{i} \mathcal{A}_{i}<0 \\
\mathcal{A}_{i+1}^{T} P_{i+1}+P_{i+1} \mathcal{A}_{i+1}<0, \\
(3) & {\left[\begin{array}{cc}
\bar{P}_{i}+\bar{\lambda} \mathcal{C}^{T} \mathcal{C}-P_{i+1} & Q_{12} \\
Q_{12}^{T} & Q_{22}
\end{array}\right]>0} \\
Q_{12} & =-\bar{P}_{i}\left(\mathcal{A}_{i}^{-1} \mathcal{B}_{i} \rho_{i}\right)-\mathcal{C}^{T} \beta_{i} \bar{\lambda}+P_{i+1}\left(\mathcal{A}_{i+1}^{-1} \mathcal{B}_{i+1} \rho_{i+1}\right) \\
Q_{22} & =1-\bar{\tau}+\bar{\lambda}\left(\beta_{i}^{2}-\gamma^{2}\right)+\left(\mathcal{A}_{i}^{-1} \mathcal{B}_{i} \rho_{i}\right)^{T} \bar{P}_{i}\left(\mathcal{A}_{i}^{-1} \mathcal{B}_{i} \rho_{i}\right) \\
& -\left(\mathcal{A}_{i+1}^{-1} \mathcal{B}_{i+1} \rho_{i+1}\right)^{T} P_{i+1}\left(\mathcal{A}_{i+1}^{-1} \mathcal{B}_{i+1} \rho_{i+1}\right),
\end{array}
$$$$
Q_{22}=1-\bar{\tau}+\bar{\lambda}\left(\beta_{i}^{2}-\gamma^{2}\right)+\left(\mathcal{A}_{i}^{-1} \mathcal{B}_{i} \rho_{i}\right)^{T} \bar{P}_{i}\left(\mathcal{A}_{i}^{-1} \mathcal{B}_{i} \rho_{i}\right)
$$ 
$\exists \bar{\tau}>0, \bar{\lambda}>0$, and $\bar{P}_{i}=\bar{\tau}_{i} P_{i}$ and $P_{i+1}$ are real symmetric positive definite matrices.

Proof. Introducing a new state variable $\bar{y}_{i}=y-\mathcal{A}_{i}^{-1} \mathcal{B}_{i} \rho_{i}$, so that the $i^{\text {th }}$ dynamic equation becomes $\dot{\bar{y}}_{i}=\mathcal{A}_{i} \bar{y}_{i}$. Define a set enclosed by an ellipsoid as $\Omega_{i} \in\left\{\bar{y}_{i}: \bar{y}_{i}^{T} P_{i} \bar{y}_{i} \leq 1\right\}$ where $P_{i}$ is some real symmetric positive-definite matrix. Suppose the ellipsoid set $\Omega_{i}$ encloses either the starting point of the trajectory or the point where the trajectory crosses some previous manifold $\mathcal{C}_{i-1} y=\beta_{i-1}$. If the ellipsoid set $\Omega_{i}$ is invariant, then by definition, the trajectory will remain within $\Omega_{i}$ for the duration that it is governed by the $i^{\text {th }}$ dynamic equation, that is, until the trajectory crosses the manifold $\mathcal{C}_{i} y=\beta_{i}$. After crossing the manifold, the system is governed by a different dynamic equation, and the invariance no longer applies. The ellipsoid set $\Omega_{i}$ is invariant for the $i^{t h}$ dynamic equation if the inequality,

$$
\mathcal{A}_{i}^{T} P_{i}+P_{i} \mathcal{A}_{i}<0
$$

is satisfied. The inequality is the derivative of the Lyapunov function $V=$ $\bar{y}_{i}^{T} P_{i} \bar{y}_{i}$. Introducing a positive scalar variable $\bar{\tau}$ and defining $\bar{P}_{i}=\bar{\tau} P_{i}$. The significance of $\bar{\tau}$ will be addressed later. Condition 1 is obtained by multiplying boths sides of (16) by $\bar{\tau}$ and substituting $\bar{P}_{i}$.

Let us define a sub-set $\dot{\Omega}_{i} \in \Omega_{i}$ as,

$$
\dot{\Omega}_{i} \in\left\{\begin{array}{c}
y:\left(y-\mathcal{A}_{i}^{-1} \mathcal{B}_{i} \rho_{i}\right)^{T} P_{i}\left(y-\mathcal{A}_{i}^{-1} \mathcal{B}_{i} \rho_{i}\right) \leq 1 \\
\left(-\beta_{i}+y^{T} \mathcal{C}_{i}^{T}\right)\left(\mathcal{C}_{i} y-\beta_{i}\right)<\gamma^{2}
\end{array}\right\},
$$

where $\gamma>0$ is some small parameter that defines a neighbourhood about the manifold $C_{i} y=\beta_{i}$. Using the $\mathcal{S}$-procedure, a new inequality is defined for all $y \notin \Omega_{i}$,

$$
\begin{gathered}
y^{T}\left(P_{i}+\lambda \mathcal{C}_{i}^{T} \mathcal{C}_{i}\right) y-2\left(\lambda \beta_{i} \mathcal{C}_{i}+\left(\mathcal{A}_{i}^{-1} \mathcal{B}_{i} \rho_{i}\right) P_{i}\right) y \\
+\left(\mathcal{A}_{i}^{-1} \mathcal{B}_{i} \rho_{i}\right)^{T} P_{i}\left(\mathcal{A}_{i}^{-1} \mathcal{B}_{i} \rho_{i}\right)-1-\lambda\left(\gamma^{2}-\beta_{i}^{2}\right)>0
\end{gathered}
$$

$\exists \lambda>0$. We now introduce a new state variable $\bar{y}_{i+1}=y-\mathcal{A}_{i+1}^{-1} \mathcal{B}_{i+1} \rho_{i+1}$ such that the $(i+1)^{t h}$ dynamic equation becomes $\dot{\bar{y}}_{i+1}=\mathcal{A}_{i+1} \bar{y}_{i+1}$. As before, we define an ellipsoid set $\Omega_{i+1} \in\left\{\bar{y}_{i+1}: \bar{y}_{i+1}^{T} P_{i+1} \bar{y}_{i+1} \leq 1\right\}$ where $P_{i+1}$ is a real symmetric positive definite matrix. The ellipsoid set $\Omega_{i+1}$ is invariant if $P_{i+1}$ satisfies,

$$
\mathcal{A}_{i+1}^{T} P_{i+1}+P_{i+1} \mathcal{A}_{i+1}<0 .
$$

If a trajectory, governed by the $(i+1)^{t h}$ dynamic equation, starts within or enters the ellipsoidal set $\Omega_{i+1}$, and that set is invariant, then the trajectory will be enclosed by the ellipsoidal set $\Omega_{i+1}$ for the duration that it is governed by the $(i+1)^{t h}$ dynamic equation, that is, until the next manifold is crossed. Thus condition 2 is derived.

If the $\dot{\Omega}_{i} \subset \Omega_{i+1}$, it follows that any trajectory governed by the $i^{\text {th }}$ dynamic equation, that starts within an invariant ellipsoid set $\bar{y}_{i}^{T} P_{i} \bar{y}_{i} \leq 1$, will enter the ellipsoid set $\bar{y}_{i+1}^{T} P_{i+1} \bar{y}_{i+1} \leq 1$ as it crosses the manifold. If the second ellipsoid 
set is invariant, then the trajectory will be enclosed by that set until it reaches the next manifold. Thus, the trajectory is enclosed to one of a succession of invariant ellipsoids that change as the trajectory crosses each manifold.

The union of all the sets bounded between the manifolds and the edge of the ellipsoid sets, form a limit on the trajectory of the switching system, as shown in figure 1 . Using the $\mathcal{S}$-procedure, equation (17) and the inequality $\bar{y}_{i+1}^{T} P_{i+1} \bar{y}_{i+1} \leq 1$, a new condition for $\Omega_{i} \subset \Omega_{i+1}$ is derived,

$$
\begin{gathered}
y^{T}\left(P_{i}+\lambda \mathcal{C}_{i}^{T} \mathcal{C}_{i}\right) y-2\left(\lambda \beta_{i} \mathcal{C}_{i}+\left(\mathcal{A}_{i}^{-1} \mathcal{B}_{i} \rho_{i}\right)^{T} P_{i}\right) y, \\
+\left(\mathcal{A}_{i}^{-1} \mathcal{B}_{i} \rho_{i}\right)^{T} P_{i}\left(\mathcal{A}_{i}^{-1} \mathcal{B}_{i} \rho_{i}\right)+\tau-1+\lambda\left(\beta_{i}^{2}-\gamma^{2}\right), \\
-\tau\left(y-\mathcal{A}_{i+1}^{-1} \mathcal{B}_{i+1} \rho_{i+1}\right)^{T} P_{i+1}\left(y-\mathcal{A}_{i+1}^{-1} \mathcal{B}_{i+1} \rho_{i+1}\right)>0 .
\end{gathered}
$$

$\exists \tau>0, \lambda>0$. Denote $\bar{\tau}=1 / \tau, \bar{P}_{1}=P_{1} / \tau$, and $\bar{\lambda}=\lambda / \tau$, then, the inequality can be expressed as the linear matrix inequality (LMI) in condition 3.

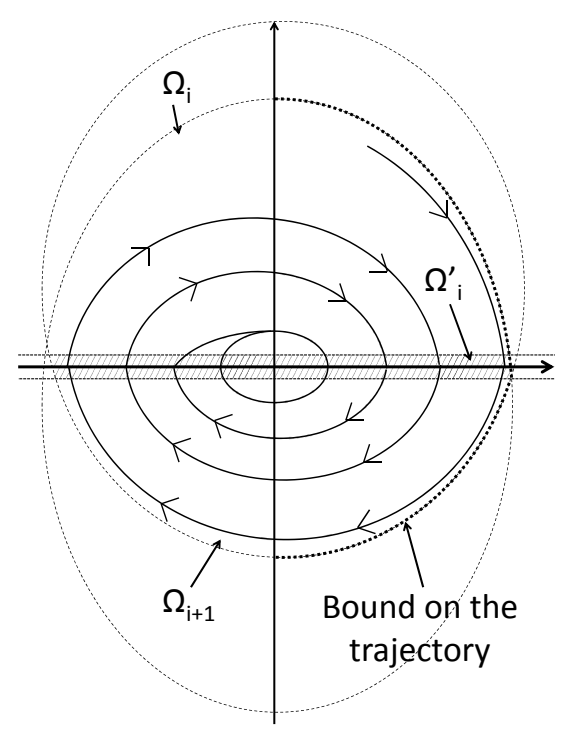

Fig. 1: Trajectory bounded by succession of invariant ellipsoids

We now outline a general procedure for obtaining the maximum output bound based on theorem 1. Denote $\sigma=\chi y$, where $\chi \in \mathbb{R}^{1 \times n}$, as the output function. Our intention is to obtain the maximum bound of $\sigma$. Introducing $\bar{y}_{i+1}^{T} \chi^{T} \chi \bar{y}_{i+1}<\epsilon$ where $\epsilon$ is a bound on the output. In our original coordinate 
system $y$, the maximum output bound in terms of $\epsilon$ is,

$$
\sigma_{\text {Max }}=\operatorname{sgn}\left(\sigma_{\operatorname{Max}}\right) \sqrt{\epsilon}+\chi \mathcal{A}_{i+1}^{-1} \mathcal{B}_{i+1} \rho_{i+1} \geq \chi y
$$

We seek the minimum $\epsilon$ such that,

$$
\bar{y}_{i+1}^{T} P_{i+1} \bar{y}_{i+1}>\bar{y}_{i+1}^{T} \chi^{T}(1 / \epsilon) \chi \bar{y}_{i+1}
$$

i.e. the output bound is within the second invariant ellipsoid. For $i=1$, the objective can be redefined in the form of the following convex LMI optimization problem: For some positive definite symmetrical $\bar{P}_{1}, P_{2} \in \mathbb{R}^{n \times n}$, minimize $\epsilon$ such that:

$$
\begin{aligned}
& {\left[\begin{array}{cc}
P_{2} & \chi^{T} \\
\chi & \epsilon
\end{array}\right] \geq 0} \\
& \left(y-\mathcal{A}_{1}^{-1} \mathcal{B}_{1} \rho_{1}\right)^{T} P_{1}\left(y-\mathcal{A}_{1}^{-1} \mathcal{B}_{1} \rho_{1}\right) \leq \bar{\tau}, \\
& \mathcal{A}_{1}^{T} \bar{P}_{1}+\bar{P}_{1} \mathcal{A}_{1}<0 \text {, } \\
& \mathcal{A}_{2}^{T} P_{2}+P_{2} \mathcal{A}_{2}<0 \\
& {\left[\begin{array}{cc}
\bar{P}_{1}+\bar{\lambda} \mathcal{C}^{T} \mathcal{C}-P_{2} & Q_{12} \\
Q_{12}^{T} & Q_{22}
\end{array}\right]>0,} \\
& Q_{12}=-\bar{P}_{1}\left(\mathcal{A}_{1}^{-1} \mathcal{B}_{1} \rho_{1}\right)-\mathcal{C}^{T} \beta_{1} \bar{\gamma}+P_{2}\left(\mathcal{A}_{2}^{-1} \mathcal{B}_{2} \rho_{2}\right), \\
& Q_{22}=1-\bar{\tau}+\bar{\lambda}\left(\beta_{1}^{2}-\gamma^{2}\right)+\left(\mathcal{A}_{1}^{-1} \mathcal{B}_{1} \rho_{1}\right)^{T} P_{1}\left(\mathcal{A}_{1}^{-1} \mathcal{B}_{1} \rho_{1}\right) \text {, } \\
& -\left(\mathcal{A}_{2}^{-1} \mathcal{B}_{2} \rho_{2}\right)^{T} P_{2}\left(\mathcal{A}_{2}^{-1} \mathcal{B}_{2} \rho_{2}\right)
\end{aligned}
$$

$\exists \bar{\tau}>0, \bar{\lambda}>0$ where step 1 is the Schur complement of equation (21), and steps 2-5 are obtained from theorem 1 .

The state-trajectory of the system may cross multiple switching manifolds before reaching the maximum output. Successive invariant ellipsoids for $i \geq 2$ are obtained by successive use of the following convex LMI optimization: For some positive symmetrical $P_{i+1} \in \mathbb{R}^{n \times n}$, and a known $P_{i}$ - which is obtained from the previous optimization - minimize $\epsilon$ such that:

$$
\begin{gathered}
Q_{12}=-\bar{P}_{i}\left(\mathcal{A}_{i}^{-1} \mathcal{B}_{i} \rho_{i}\right)-\mathcal{C}_{i}^{T} \beta_{i} \bar{\lambda}+P_{i+1}\left(\mathcal{A}_{i+1}^{-1} \mathcal{B}_{i+1} \rho_{i+1}\right) \\
Q_{22}=1-\bar{\tau}+\bar{\lambda}\left(\beta_{i}^{2}-\gamma^{2}\right)+\left(\mathcal{A}_{i}^{-1} \mathcal{B}_{i} \rho_{i}\right)^{T} \bar{P}_{i}\left(\mathcal{A}_{i}^{-1} \mathcal{B}_{i} \rho_{i}\right) \\
-\left(\mathcal{A}_{i+1}^{-1} \mathcal{B}_{i+1} \rho_{i+1}\right)^{T} P_{i+1}\left(\mathcal{A}_{i+1}^{-1} \mathcal{B}_{i+1} \rho_{i+1}\right)
\end{gathered}
$$

$\exists \bar{\tau}>0, \bar{\lambda}>0$ 
We consider the application of this procedure to finding the maximum output bound of systems controlled by the 2-sliding mode controllers. Any linear system of the form,

$$
\dot{x}=A x+B u(t), \quad \sigma=S x,
$$

controlled by either the twisting controller or generalized sub-optimal controller, can be expressed as the switching system in theorem 1. Thus, we can use the outlined procedure to obtain the maximum output bound. The homogeneous controller has a non-linear switching manifold and requires a modification to the procedure before it can be used.

1) The twisting controller has two switching manifolds, $\sigma=0$ and $\dot{\sigma}=0$ and four possible $\rho_{i}$; one for each quadrant of the state-space $\{\sigma, \dot{\sigma}\}$, i.e.

$$
\begin{aligned}
& \left\{\begin{array}{c|c}
\rho_{i}=r_{1}+r_{2} & \sigma>0 \\
\rho_{i+1}=r_{1}-r_{2} & \dot{\sigma}>0
\end{array}\right\}, \quad\left\{\begin{array}{c|c}
\rho_{i}=-r_{1}+r_{2} & \sigma<0 \\
\rho_{i+1}=r_{1}+r_{2} & \dot{\sigma}>0
\end{array}\right\}, \\
& \left\{\begin{array}{c|c}
\rho_{i}=-r_{1}-r_{2} & \sigma<0 \\
\rho_{i+1}=-r_{1}+r_{2} & \dot{\sigma}<0
\end{array}\right\}, \quad\left\{\begin{array}{c|c}
\rho_{i}=r_{1}-r_{2} & \sigma>0 \\
\rho_{i+1}=-r_{1}-r_{2} & \dot{\sigma}<0
\end{array}\right\} \text {. }
\end{aligned}
$$

2) The generalized sub-optimal controller has a switching manifold that moves proportionally with the peak oscillations of the output sliding variable, that is $\sigma=r_{2} \sigma_{M}$, where $\sigma_{M}$ is the previous extremal value of the oscillating $\sigma$. The controller has two possible $\rho_{i}$; they are $\rho_{i}=r_{1}$, and $\rho_{i+1}=-r_{1}$ and the reverse.

3) The homogeneous controller has a non-linear switching manifold, and thus theorem 1 cannot be directly applied. Denote $\Sigma(y)=0$ as the nonlinear manifold. We derive a new condition which can be used to guarantee that the set $\Omega_{i+1}$ encloses the intersection of the set $\Omega_{i}$ and the manifold $\Sigma(y)$ between zero and some maximum upper-bound $\widetilde{\sigma}$. For simplicity, we consider the case where the trajectory begins in the half-plane $\sigma>0$. Define,

$$
\sigma=\chi_{1} y, \quad \dot{\sigma}=\chi_{2} y, \quad \text { and } \quad \chi=\left[\begin{array}{ll}
\chi_{1} & \chi_{2}
\end{array}\right]^{T} .
$$

Let us denote $\widetilde{\Omega}_{i}$ as a new elliptical set,

$$
\widetilde{\Omega}_{i} \in\left\{y: 0<\chi_{1} y<\tilde{\sigma},(\chi y-E)^{T} \widetilde{P}(\chi y-E)<1\right\},
$$

where $E=\left[\begin{array}{ll}1 / \sqrt{P_{11}} & 0\end{array}\right]^{T}, \widetilde{P} \in \mathbb{R}^{2 \times 2}$ is,

$$
\widetilde{P}=\left[\begin{array}{cc}
P_{11} & 0 \\
0 & \left(2 \sqrt{P_{11}}-P_{11} \tilde{\sigma}\right) / r_{2}^{2}
\end{array}\right] \quad P_{11}>0,
$$

and $\tilde{\sigma}$ is an upper-bound on the sliding variable output. We find by definition of $\widetilde{\Omega}_{i}$, that it is an ellipsoid which encloses the switching manifold $\Sigma(y)=0$ upto $\sigma=\tilde{\sigma}$. The set $\dot{\Omega}_{i}$ in theorem 1 is then replaced by $\dot{\Omega}_{i} \in\left\{\widetilde{\Omega}_{i} \cap \Omega_{i}\right\}$, and a new 
inequality proposed such that $y \notin \Omega_{i}$,

$$
\begin{aligned}
& y^{T}\left(P_{1}+\lambda \chi^{T} \widetilde{P} \chi-P_{2}\right) y-2\left(P_{1}\left(\mathcal{A}^{-1} \mathcal{B} r_{1}\right)+\lambda \chi^{T} \widetilde{P} E\right) y, \\
& +\left(\mathcal{A}^{-1} \mathcal{B}\right)^{T} P_{1}\left(\mathcal{A}^{-1} \mathcal{B}\right)-1-\lambda+\lambda E^{T} \widetilde{P} E>0 .
\end{aligned}
$$

A new LMI is derived by following the procedure in the proof of theorem 1, but with (17) replaced by (24):

$$
\begin{aligned}
& {\left[\begin{array}{cc}
\bar{P}_{1}+\bar{\lambda} \chi^{T} \widetilde{P} \chi-P_{2} & Q_{12} \\
Q_{12}^{T} & Q_{22}
\end{array}\right]>0} \\
& Q_{12}=-\bar{P}_{1}\left(\mathcal{A}^{-1} \mathcal{B} r_{1}\right)-\bar{\lambda} \mathcal{C}^{T} \widetilde{P} E-P_{2}\left(\mathcal{A}^{-1} \mathcal{B} r_{1}\right) \\
& Q_{22}=1-\bar{\tau}+\left(\mathcal{A}^{-1} \mathcal{B} r_{1}\right)^{T} \bar{P}_{1}\left(\mathcal{A}^{-1} \mathcal{B} r_{1}\right) \\
& -\left(\mathcal{A}^{-1} \mathcal{B} r_{2}\right) P_{2}\left(\mathcal{A}^{-1} \mathcal{B} r_{2}\right)
\end{aligned}
$$

$\exists \bar{\tau}>0, \bar{\lambda}>0$. The maximum output bound is $-\sqrt{\epsilon}-\chi_{1} \mathcal{A}^{-1} \mathcal{B} r_{1}-1$, where $\epsilon$ is obtained by following the general outlined procedure but with step 5 replaced by the new LMI. For systems starting in the half-plane $\sigma<0$, the following parameters are replaced: $E=-E, r_{1}=-r_{1}$.

\section{Example}

The performance estimation procedures are illustrated with an example second order system with actuator and sensor parasitic dynamics. Introducing a system with second order plant, the sliding variable as output, and a set of fast-acting first order dynamics acting on the actuator and sensors,

$$
\begin{aligned}
& {\left[\begin{array}{c}
\dot{z} \\
\ddot{z} \\
0.03 \dot{x} \\
0.03 \ddot{x} \\
0.01 \dot{\tilde{u}}
\end{array}\right]=\left[\begin{array}{ccccc}
0 & 1 & 0 & 0 & 0 \\
-15 & -5 & 0 & 0 & 3 \\
1 & 0 & -1 & 0 & 0 \\
0 & 1 & 0 & -1 & 0 \\
0 & 0 & 0 & 0 & -1
\end{array}\right]\left[\begin{array}{c}
z \\
\dot{z} \\
x \\
\dot{x} \\
\tilde{u}
\end{array}\right]+\left[\begin{array}{l}
0 \\
0 \\
0 \\
0 \\
1
\end{array}\right] u(\sigma),} \\
& \sigma=10 x+\dot{x}
\end{aligned}
$$

The described procedures are used to predict chatter amplitude, settling time, and maximum output bound of this system. Chattering amplitude was found directly from the frequency response (Nyquist plots) of the system. We first set $\zeta=0$ in the describing functions $N(a, \omega, \zeta=0)$. Placing equation (9) in the frequency domain $(p=j \omega)$ we have,

$$
-\frac{1}{N(a, \omega)}=\frac{B(j \omega)}{A(j \omega)}
$$

from which we can solve for $a, \omega$. This can be done graphically on the Nyquist plot by finding the point of intersection between the frequency response and the negative inverse of the describing function. 
Settling times were found by calculating $\zeta$ and $\omega$ at a range of discrete amplitudes between $a=1$ and $a=0.2$ at intervals of $\Delta a=0.005$, e.g. $a=$ $1,0.995,0.99, \ldots$. The values of $\zeta$ and $\omega$ at some $a$ were calculated from the following optimisation problem,

$$
\min _{\zeta, \omega} \operatorname{Re}(H(a, \zeta, \omega))^{2}+\operatorname{Im}(H(a, \zeta, \omega))^{2} \quad \text { s.t. } \quad \begin{gathered}
\zeta<0 \\
\omega>0
\end{gathered},
$$

where $H(a, \zeta, \omega)=A(\zeta+j \omega)+B(\zeta+j \omega) N(a, \zeta, \omega)$. The additional constraints on $\zeta, \omega$ are added as a practical measure to ensure the solution does not fall into a false minimum. Finally, settling time is obtained by numerical integration of the values of $\zeta$ at each discrete division of $a$ to find a solution to (12).

The LMI problems for maximum output bound were solved using the Matlab LMI toolbox (MathWorks Inc, USA). The variable $\gamma$ in the LMI problem is chosen by trial and error to be $\gamma=0.0001$. The results were not found to be overly sensitive to this parameter, provided $\gamma$ was chosen to be sufficiently small.

The system is simulated using Simulink (MathWorks Inc, USA) with each of the controllers. The response of the output sliding variable $\sigma$ is shown in figure 5. The predicted maximum output bound computed using the LMI method is shown in the figure for each of the controllers. The approximated chattering amplitude and settling time (decay-rate) are also shown in figures a) and c), computed using the describing function of the controller.

The predicted maximum output bound is found to be a conservative estimate. This is further illustrated using the following second-order system with variable stiffness parameter $K$,

$$
\left[\begin{array}{c}
\dot{\sigma}(t) \\
\ddot{\sigma}(t)
\end{array}\right]=\left[\begin{array}{cc}
0 & 1 \\
K & -1
\end{array}\right]\left[\begin{array}{c}
\sigma(t) \\
\dot{\sigma}(t)
\end{array}\right]+\left[\begin{array}{l}
0 \\
1
\end{array}\right] u(\sigma), \quad \begin{array}{ll}
\sigma(0) & =1 \\
\dot{\sigma}(0) & =0
\end{array}
$$

The maximum output bound ellipsoids computed by the proposed LMI method for each of the controllers over varying values of $K=-15, K=-50$ and $K=-300$ are shown in figures 3,4 and 5 . The result is confirmed. In each case, the invariant ellipsoid produced a conservative estimate for the maximum output bound.

\section{Conclusion}

The comprehensive formalization of tuning procedures for chatter suppression sliding mode control is necessary to encourage their wider use in real systems. This papers contribution is towards this goal, by establishing straightforward procedures to estimate key performance metrics of some common chatter suppression 2-sliding mode controllers.

Describing functions are derived to estimate instantaneous frequency and damping during the transient phase. These are used to find settling times between an initial starting amplitude and some final amplitude, and to find the chattering amplitude under steady-state conditions. Both procedures are found to be accurate in an example system which satisfies the low-pass filter 
assumption. This assumption, in general, is not overly restrictive and is true for a wide range of systems. A procedure based on invariant ellipsoids is presented to estimate the maximum output bound. This method computes a sequential series of invariant ellipsoids which enclose the state-trajectory of the 2-sliding mode controlled system. A set of linear matrix inequalities (LMIs) are derived to compute the ellipsoids sets. Optimal solutions are readily found using standard LMI methods.

\section{References}

[1] Derek Atherton. Stability of nonlinear systems. Research Studies Press, Chichester, 1981.

[2] G. Bartolini, A. Pisano, E. Punta, and E. Usai. A survey of applications of second-order sliding mode control to mechanical systems. International Journal of Control, 76(9-10):875-892, 2003.

[3] F. Blanchini. Set invariance in control. Automatica, 35(11):1747-1767, 1999.

[4] I. Boiko. Analysis of closed-loop performance and frequency-domain design of compensating filters for sliding mode control systems. IEEE Transactions on Automatic Control, 52(10):1882-1891, 2007.

[5] I. Boiko, L. Fridman, and M. L. Castellanos. Analysis of second-order sliding-mode algorithms in the frequency domain. IEEE Transactions on Automatic Control, 49(6):946-950, 2004.

[6] I. Boiko, L. Fridman, A. Pisano, and E. Usai. Analysis of chattering in systems with second-order sliding modes. IEEE Transactions on Automatic Control, 52(11):2085-2102, 2007.

[7] Stephen P. Boyd. Linear matrix inequalities in system and control theory. Society for Industrial and Applied Mathematics, Philadelphia, Pa., 1994.

[8] F. Dinuzzo and A. Ferrara. Higher order sliding mode controllers with optimal reaching. Automatic Control, IEEE Transactions on, 54(9):2126 -2136 , sept. 2009.

[9] Christopher Edwards and Sarah K. Spurgeon. Sliding mode control : theory and applications. Taylor and Francis, London, 1998.

[10] A. Levant. Sliding order and sliding accuracy in sliding mode control. International Journal of Control, 58(6):1247-1263, 1993.

[11] A. Levant. Higher-order sliding modes, differentiation and output-feedback control. International Journal of Control, 76(9-10):924-941, 2003.

[12] A. Levant. Principles of 2-sliding mode design. Automatica, 43(4):576-586, 2007. 
[13] P. Li and Z.-Q. Zheng. Robust adaptive second-order sliding-mode control with fast transient performance. Control Theory Applications, IET, 6(2):305-312, 192012.

[14] Jean-Jacques E. Slotine and Weiping Li. Applied nonlinear control. Prentice Hall, Englewood Cliffs, N.J., 1991. Jean-Jacques E. Slotine, Weiping Li.

[15] T. Thibodeau, W. C. Tong, and T. S. Hu. Set invariance and performance analysis of linear systems via truncated ellipsoids. Automatica, 45(9):20462051, 2009.

[16] V. I. Utkin, J. Guldner, and J. Shi. Sliding mode control in electromechanical systems. Taylor and Francis, London, 1999.

[17] Z. Vukic. Nonlinear control systems. Dekker, New York, 2003.

[18] Q. Zong, Z.-S. Zhao, and J. Zhang. Brief paper: Higher order sliding mode control with self-tuning law based on integral sliding mode. Control Theory Applications, IET, 4(7):1282 -1289, july 2010. 


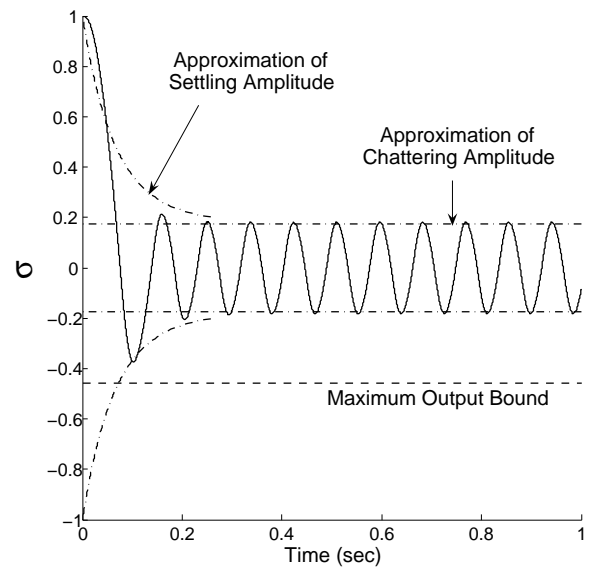

(a) Twisting controller

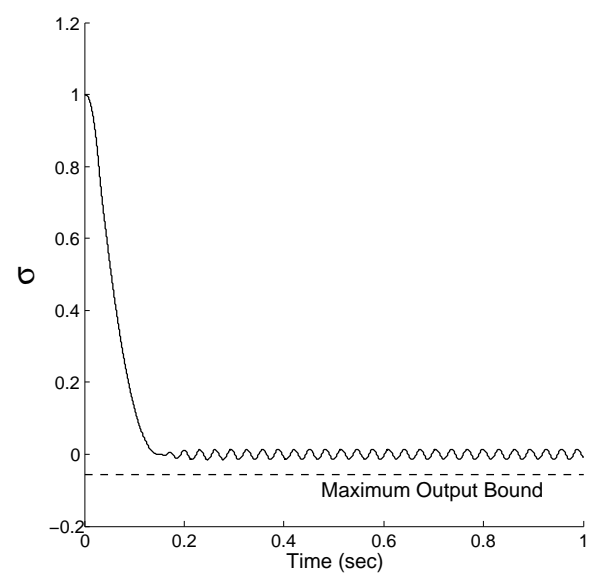

(b) Homogeneous controller

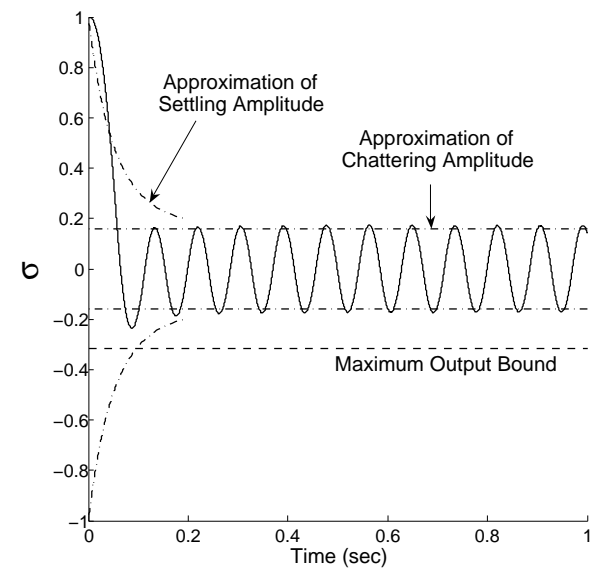

(c) Generalized sub-optimal controller

Fig. 2: Output response and predicted performance of the system in (26) 


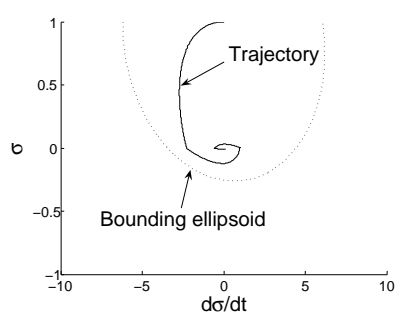

(a) $K=-15$

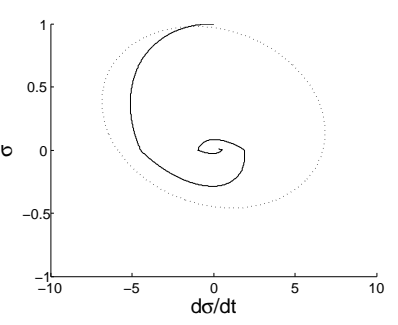

(b) $K=-50$

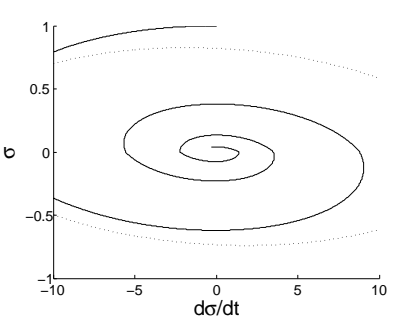

(c) $K=-300$

Fig. 3: Trajectory and approximate bounded ellipsoid $(\sigma, \dot{\sigma}) P_{2}(\sigma, \dot{\sigma})^{T}=1$ of system (28) controlled by twisting controller

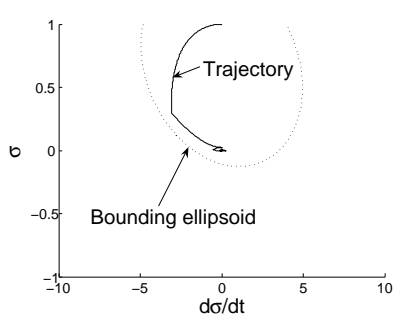

(a) $K=-15$

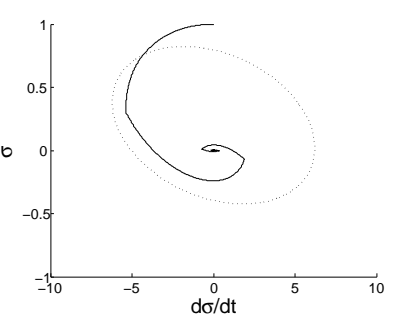

(b) $K=-50$

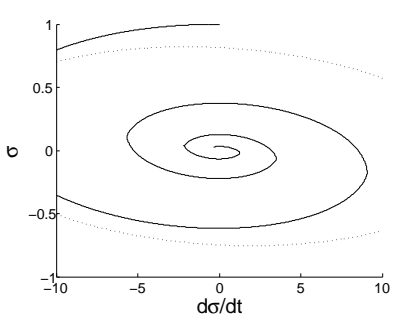

(c) $K=-300$

Fig. 4: Trajectory and approximate bounded ellipsoid $(\sigma, \dot{\sigma}) P_{2}(\sigma, \dot{\sigma})^{T}=1$ of system (28) controlled by generalized sub-optimal controller

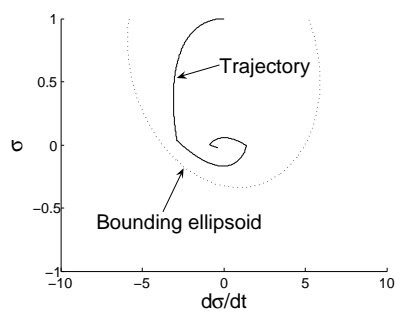

(a) $K=-15$

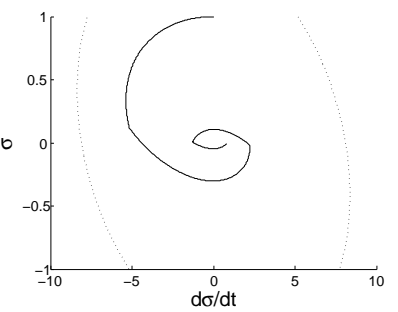

(b) $K=-50$

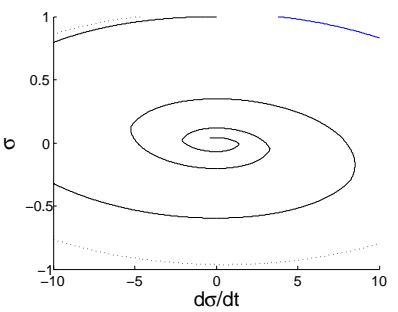

(c) $K=-300$

Fig. 5: Trajectory and approximate bounded ellipsoid $(\sigma, \dot{\sigma}) P_{2}(\sigma, \dot{\sigma})^{T}=1$ of system (28) controlled by homogeneous controller 